\title{
Managed Care in der Schweiz: Wo stehen wir?
}

Managed Care bezeichnet die Summe aller Steuerund Integrationsimpulse im Gesundheitswesen mit dem Ziel, eine qualitäts- und kosteneffektive medizinische Versorgung zu fördern [1]. Der Begriff «Managed Care» wird im englischen Sprachraum kaum mehr benutzt, vielmehr spricht man von «integrierter Versorgung». Die WHO definiert integrierte Versorgung als «a concept bringing together inputs, delivery, management and organization of services related to diagnosis, treatment, care, rehabilitation and health promotion. Integration is a means to improve services in relation to access, quality, user satisfaction and efficiency».

In der Schweiz wurden die ersten experimentellen Managed-Care-Erfahrungen zu Beginn der neunziger Jahre in Zürich und Genf gemacht: Das Krankenversicherungsgesetz (KVG) war noch nicht in Kraft, und im alten Kranken- und Unfallversicherungsgesetz (KUVG) waren diese alternativen Versicherungsmodelle noch nicht zugelassen. Mit dem Inkrafttreten des KVG am 1. Januar 1996 ist es gesetzlich möglich, solche Versicherungsformen anzubieten. Ausgehend vom Subsidiaritätsprinzip unseres liberalen Staates können die Vertragspartner, also Ärzte und Versicherer, solche Modelle entwickeln. Viele setzen grosse Hoffnungen in die Steuerungswirkung solcher integrierter Netze. Was hat sich seit 1996 getan?

\section{Bis heute haben sich in der Schweiz 86 Ärztenetze, oft unter Initiative der Ärzteschaft, gebildet}

Peter Berchtold, der Präsident des Forums Managed Care [2], schildert in dieser Ausgabe der Schweizerischen Ärztezeitung auf Seite 2038 die Entwicklung der Ärztenetze: Bis heute haben sich in der Schweiz 86 ziemlich unterschiedliche Ärztenetze, oft unter Initiative der Ärzteschaft, gebildet. In gewisse Regionen haben bis $\mathrm{zu}$ 36\% (Thurgau) der Versicherten diese Versicherungsformen gewählt [3]. Durchschnittlich engagieren sich 47\% aller Grundversorgerinnen und Grundversorger in Ärztenetzen.

Die FMH bewertet diese Entwicklung als äusserst positiv: Als Standesorganisation von rund 33000 Ärztinnen und Ärzten hat sie bereits im Jahr 2006 ein Thesenpapier veröffentlicht, das die Eckwerte für die Entwicklung von Managed Care in der Schweiz darstellt [4].

\section{Staatliche Anreize zur Entwicklung von Ärztenetzen sind aus Sicht der FMH durchaus prüfenswert}

Wie ist nun diese Entwicklung zu bewerten - genügt sie oder soll der Staat unterstützend eingreifen? Der Bundesrat hat im Jahr 2004 eine Botschaft verabschiedet, in der er die Entwicklung von Managed Care in der Schweiz fördern will (Vorlage 04.062). Diese wurde bereits in der Wintersession 2006 im Ständerat verabschiedet und liegt heute in der nationalrätlichen Kommission. Unsere Kommission hat bereits im Sommer 2004 beschlossen (und inzwischen noch einmal bestätigt), dass sie die Vorlage Managed Care gemeinsam mit der Vorlage zur Kostenbeteiligung (04.034 - vom Ständerat bereits in der Herbstsession 2004 angenommen) und zur Vertragsfreiheit (04.032) behandeln will. Letztere ist immer noch bei der ständerätlichen Kommission, die daran ist, eine Nachfolgeregelung für den Zulassungsstopp zu beraten. Da für dieses Thema wahrscheinlich noch viele Diskussionen nötig sind, wird die Vorlage Managed Care in unserer Kommission noch für einige Zeit blockiert bleiben. Staatliche Anreize zur Entwicklung von Ärztenetzen sind in unseren Augen durchaus prüfenswert; angesichts der geschilderten Umstände wird dies wohl kaum vor 2010 möglich sein. Aber eben, auch ohne staatlichen Interventionismus setzt sich das Modell der Ärztenetze erfreulicherweise langsam durch.

Dr. med. Ignazio Cassis, MPH, Vizepräsident der FMH und Nationalrat

1 Berchtold P. Forum Managed Care. Newsletter Public Health Schweiz. 2008;7(3):10.

2 Das Forum Managed Care ist ein offener Verein für die Diskussion und Verbreitung innovativer Systeme im Schweizer Gesundheitswesen. Es versteht sich als das massgebliche Forum für zukunftsfähige, qualitäts- und patientenorientierte Konzeptionen. www.fmc.ch.

3 SG 26\%, SH 25\%, AG 23\%, GR 19\%, GE 14\%, ZH 12\%.

4 FMH. Ja zu Vernetzung und Qualität - ja zu «Managed Care». Schweiz Ärztezeitung. 2006;87(36):1535-40. 